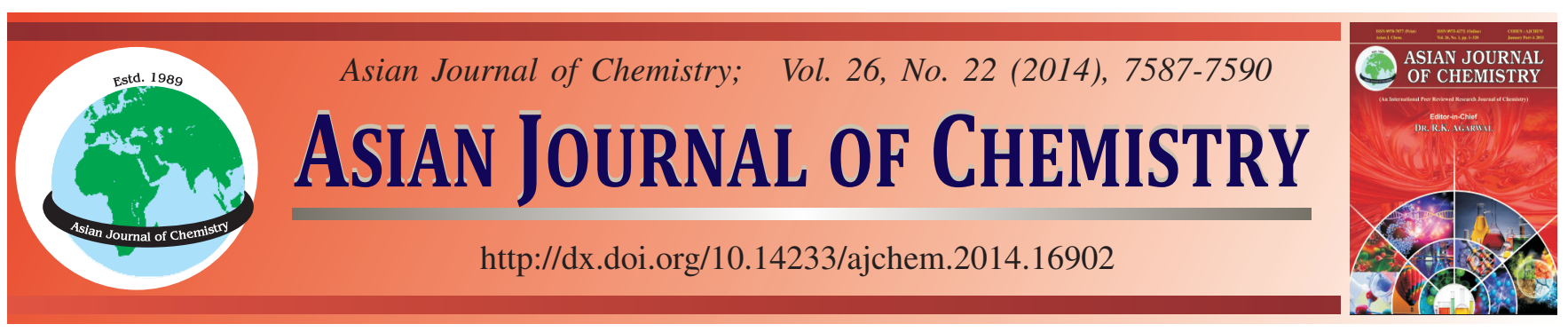

\title{
Synthesis, Characterization of 3-(Bromomethyl)-2-cyclopropyl-4-(4-fluorophenyl)quinoline and Its Crystal Structure
}

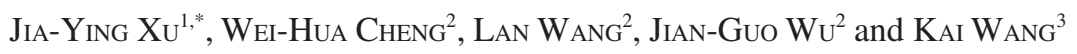

${ }^{1}$ College of Chemical and Biological Engineering, Yancheng Institute of Technology, Yancheng 224051, Jiangsu Province, P.R. China ${ }^{2}$ Department of Light Chemical Engineering, Yancheng Institute of Industry Technology, Yancheng 224051, Jiangsu Province, P.R. China ${ }^{3}$ High Technology Research Institute of Nanjing University, Changzhou, 213162, Jiangsu Province, P.R. China

*Corresponding author: E-mail: xujiaying-1984@163.com

3-(Bromomethyl)-2-cyclopropyl-4-(4-fluorophenyl)quinoline (I), an important intermediate to synthesize pitavastatin calcium. It was prepared from ethyl 2-cyclopropyl-4-(4-fluorophenyl)quinoline-3-carboxylate via reduction by $\mathrm{KBH}_{4} / \mathrm{ZnCl}_{2}$ and then bromide by $\mathrm{PBr}_{3}$. $\mathrm{The}$ product was characterized by NMR and LC-MS. The crystal structure of compound I was investigated using X-ray diffraction and SHELXTL-97 software. The result indicated that compound I crystallized in the triclinic system, space group P-1 with $\mathrm{a}=9.6150(19), \mathrm{b}=9.868(2), \mathrm{c}=$ $10.060(2) \AA, V=783.3(4) \AA^{3} ; Z 2$.

Keywords: 3-(Bromomethy)-2-cyclopropy-4-(4-fluorophenyl)quinolone, Synthesis, Characterization, Crystal structure.

\section{INTRODUCTION}

Pitavastatin calcium, a new treatment of high cholesterol statins $^{1,2}$. It is used as HMG CoA reductase inhibitor by Nissan Chem Corporation and sold in Japan in 2003. It was proved to be a kind of long duration, good tolerance and high security drug to treat hyperlipidemia and high cholesterol statins, which have a broad market prospect.

3-(Bromomethyl)-2-cyclopropyl-4-(4-fluorophenyl)quinoline(I) is widely concerned as the most important intermediate to synthesize pitavastatin calcium ${ }^{3}$. Now, some synthetic routes are reported about (I) in the literatures, such as, (I) could be prepared from 2-cyclopropyl-4-(4-fluorophenyl)-3-methylquinoline by NBS bromide in $\mathrm{MeCN}$ and $\mathrm{CCl}_{4}$. But the yield is not high. Herein, we report the synthesis of (I) from ethyl 2-cyclopropyl-4-(4-fluorophenyl)quinoline3 -carboxylate (1) with an overall yield of about $58.5 \%$. Meanwhile, the crystal structure of (I) was also investigated. The synthetic route of compound I was presented as Scheme-I.

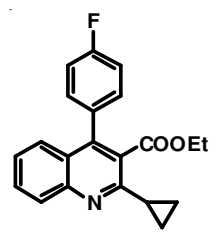

1

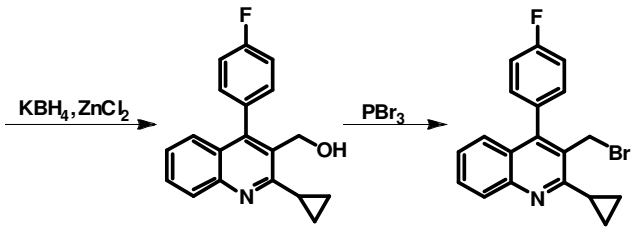

2
Scheme-I: Route for the synthesis of compound I

\section{EXPERIMENTAL}

Ethyl 2-cyclopropyl-4-(4-fluorophenyl)quinoline-3carboxylate (1) was supplied by Well Chemical Co. Ltd of Jiangsu (Yancheng, People's Republic of China), its mass content is $98.5 \%$ determined by $\mathrm{GC}$. $\mathrm{KBH}_{4}, \mathrm{ZnCl}_{2}$ and $\mathrm{PBr}_{3}$ was supplied by Sinopharm Chemical Reagent Co. Ltd of China. All other chemicals were of reagent grade and used without purification as received.

${ }^{1} \mathrm{H}$ NMR spectrum was obtained with Bruker AV-500 spectrometer at $500.13 \mathrm{MHz}$ and measured in $\mathrm{CD}_{3} \mathrm{OD}$ solution at $25 \pm 0.5^{\circ} \mathrm{C}$. The sample was dissolved in a $5 \mathrm{~mm}$ diameter tube at a concentration of $20 \mathrm{mg} / \mathrm{mL}$. X-ray diffraction was performed on a Bruker APEXII CCD diffractometer. Mass spectrum of (I) was analyzed using Trace DSQ LC/MS (Thermo Electron Co., USA).

Synthesis of compound I: In a 2 L four-necked flask, THF (30 mL), $\mathrm{ZnCl}_{2}(3.15 \mathrm{~g}, 0.023 \mathrm{~mol})$ and $\mathrm{KBH}_{4}(2.45 \mathrm{~g}$, $0.045 \mathrm{~mol}$ ) were added, stirred for $2 \mathrm{~h}$ at room tempeature. ethyl 2-cyclopropyl-4-(4-fluorophenyl)quinoline-3-carboxylate (1) (4.9 g, $0.015 \mathrm{~mol})$ was dissolved in toluene and then it was added into the reaction vessel. The reaction system was heated slowly to the refluxing temperature. After $20 \mathrm{~h}$, the system was cooled and filtered. The filter cake was washed and extracted by toluene. The organic layer was combined and washed with $0.1 \mathrm{~mol} / \mathrm{L}$ sodium hydroxide solution and saturated brine until neutral. The product was collected by 
evaporating the solvent under reduced pressure and recrystallized from a mixture of toluene. Compound $\mathbf{2}$ was obtained in the form of a white powder $\left(2.8 \mathrm{~g}, 65 \%\right.$, m.p. $\left.129-130{ }^{\circ} \mathrm{C}\right)$.

Phosphorus tribromide $(0.8 \mathrm{~mL}, 8.5 \mathrm{mmol})$ is added slowly to a solution of the compound 2 ( $1.25 \mathrm{~g}, 4.25 \mathrm{mmol})$ in dichloromethane $(30 \mathrm{~mL})$. Stirring is carried out in the ice bath for $4 \mathrm{~h}$ and $150 \mathrm{~mL}$ of water are then added. The remaining solution was washed by saturated sodium bicarbonate solution until $\mathrm{pH}$ is 8 . Product was extracted with ethyl acetate and the organic phase is separated off and dried (using $\mathrm{Na}_{2} \mathrm{SO}_{4}$ ). The product was collected by evaporating the solvent under reduced pressure and recrystallized from a mixture of ethanol. By that means, $1.35 \mathrm{~g}\left(90 \%\right.$, m.p. $\left.133-135^{\circ} \mathrm{C}\right)$ of the bromide (I) can be obtained in the form of a brown powder.

Crystals of I that suitable for X-ray diffraction were obtained by slow evaporation of 1,2-dichloroethane solution of $\mathbf{I}$.

X-ray crystallography: A colourless block-like crystal of compound (I) grown in 1,2-dichloroethane with dimensions of $0.20 \times 0.10 \times 0.10 \mathrm{~mm}$ was used for structural determination. Diffraction data were collected on a Bruker APEXII CCD diffractometer by using graphite monochromated $\mathrm{MoK}_{\alpha}$ radiation $(\lambda=0.71073 \AA)$. The structure was solved by direct methods with SHELXS-97 and refined on the $\mathrm{F}^{2}$ by full-matrix least-squares method with SHELXL-97. All non-hydrogen atoms were refined anisotropically ${ }^{4-6}$.

\section{RESULTS AND DISCUSSION}

Identification of resonance in the spectra: ${ }^{1} \mathrm{H}$ NMR and LC-MS spectra of purified compound I were presented in Fig. 1.

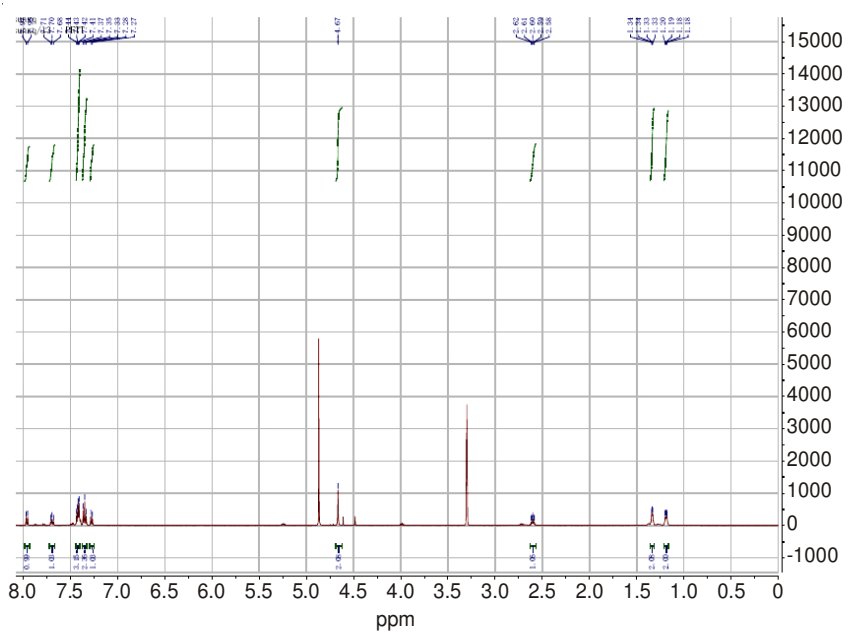

Fig. 1. ${ }^{1} \mathrm{H}$ NMR spectrum of compound I

In the ${ }^{1} \mathrm{H}$ NMR of Compound $\mathbf{I}$, the peak at $4.67 \mathrm{ppm}$ was ascribed to the proton of methylene which was substituted by atom $\mathrm{Br} .{ }^{1} \mathrm{H}$ NMR-(CD $\left.\mathrm{OD}\right): \delta 1.19(2 \mathrm{H}, \mathrm{m}), 1.33(2 \mathrm{H}, \mathrm{m})$, 2.58-2.62 (1H, m), $4.67(2 \mathrm{H}, \mathrm{s}), 7.27-7.44(6 \mathrm{H}, \mathrm{m}), 7.70(1 \mathrm{H}$, m), $7.90(1 \mathrm{H}, \mathrm{m})$.

In the LC spectrum peak at 32.619 min ascribed to the Compound I (Fig. 2). In the MS spectrum, the existence of the peaks at right end showed the Compound $\mathbf{I}, \mathrm{m} / \mathrm{z} 355.75$ was ascribed to molecular ion peak $\left(\mathrm{M}^{+}\right)$.
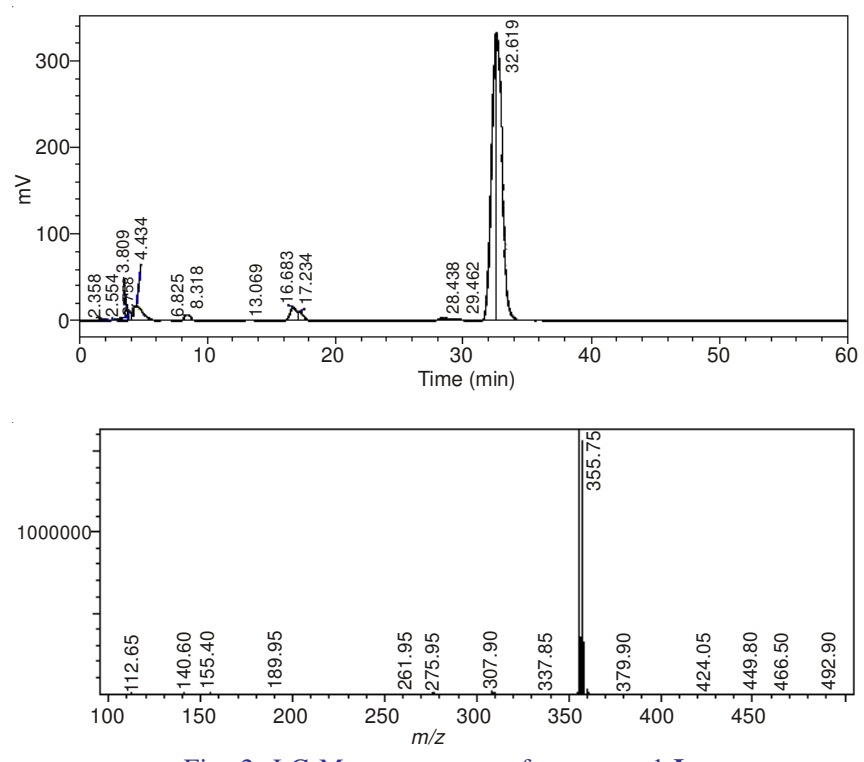

Fig. 2. LC-Mass spectrum of compound I

The crystal configuration of compound I was confirmed by X-ray structural analysis. X-ray data collection were presented in Table-1 and the geometric parameters for compound I were listed in Table-2. Molecular structure and packing plot of compound I were showed in Figs. 3 and 4, respectively.

\begin{tabular}{|c|c|}
\hline \multicolumn{2}{|c|}{$\begin{array}{c}\text { TABLE-1 } \\
\text { CRYSTALLOGRAPHIC DATA FOR COMPOUND (I) }\end{array}$} \\
\hline ITEM & Data or Description \\
\hline Formula & $\mathrm{C}_{19} \mathrm{H}_{15} \mathrm{BrFN}$ \\
\hline Formula weight & 356.22 \\
\hline Temperature $(\mathrm{K})$ & $293(2)$ \\
\hline Wavelength (̊) & 0.71073 \\
\hline Crystal system & Triclinic \\
\hline Space group & $\mathrm{P}-1$ \\
\hline $\mathrm{a}(\AA)$ & $9.6150(19)$ \\
\hline $\mathrm{b}(\AA)$ & $9.868(2)$ \\
\hline$c(\AA)$ & $10.060(2)$ \\
\hline Volume $\left(\AA^{3}\right)$ & $783.3(4)$ \\
\hline $\mathrm{Z}$ & 2 \\
\hline Calculated density $\left(\mathrm{g} / \mathrm{cm}^{3}\right)$ & 1.510 \\
\hline Absorption coefficient $\left(\mathrm{mm}^{-1}\right)$ & 2.630 \\
\hline $\mathrm{F}(000)$ & 360.0 \\
\hline Crystal size (mm) & $0.20 \times 0.10 \times 0.10$ \\
\hline Theta range for data collection $\left({ }^{\circ}\right)$ & 2.17 to 25.83 \\
\hline Reflections collected/unique & $2866 / 1420[\mathrm{R}($ int $)=0.0629]$ \\
\hline Completeness to theta $=25.38(\%)$ & 99.8 \\
\hline Max. and min. transmission & 0.621 and 0.779 \\
\hline Refinement method & Full-matrix least-squares on $\mathrm{F}^{2}$ \\
\hline Data/restraints/parameters & $1420 / 0 / 199$ \\
\hline Goodness-of-fit on $\mathrm{F}^{2}$ & 1.003 \\
\hline Final $R$ indices $[I>2 \sigma(I)]$ & $\mathrm{R} 1=0.0629, \mathrm{wR} 2=0.1679$ \\
\hline $\mathrm{R}$ indices (all data) & $\mathrm{R} 1=0.1415, \mathrm{wR} 2=0.1407$ \\
\hline Largest diff. peak and hole $\left(\mathrm{e} . \AA^{-3}\right)$ & 0.31 and -0.45 \\
\hline
\end{tabular}

According to the data from X-ray crystallographic analysis, compound $\mathbf{I}$ crystallized in a P-1 space group of the triclinic system ${ }^{7,8}$. All $\mathrm{H}$ atoms were positioned geometrically and constrained to ride on their parent atoms, with $\mathrm{C}-\mathrm{H}=0.93$ $\AA$ for aromatic $\mathrm{H}$. Other $\mathrm{H}$ atoms were positioned geometrically and refined using a riding model, with $\mathrm{C}-\mathrm{H}=0.96 \AA$ for alkyl $\mathrm{H}$, with $\operatorname{Uiso}(\mathrm{H})=1.2 \mathrm{Ueq}(\mathrm{C})$ for aromatic $\mathrm{H}$ and $\operatorname{Uiso}(\mathrm{H})$ 


\begin{tabular}{|c|c|c|c|}
\hline \multicolumn{4}{|c|}{$\begin{array}{c}\text { TABLE-2 } \\
\text { GEOMETRIC PARAMETERS FOR COMPOUND I }\end{array}$} \\
\hline Bond & Distance $(\AA)$ & Bond & Distance $(\AA)$ \\
\hline $\mathrm{Br}-\mathrm{C} 19$ & $1.958(6)$ & C8-C9 & $1.367(8)$ \\
\hline $\mathrm{N}-\mathrm{C} 4$ & $1.314(6)$ & C8-H8A & 0.9300 \\
\hline $\mathrm{N}-\mathrm{C} 5$ & $1.364(6)$ & C9-C10 & $1.394(7)$ \\
\hline $\mathrm{C} 1-\mathrm{C} 2$ & $1.422(10)$ & C9-H9A & 0.9300 \\
\hline $\mathrm{C} 1-\mathrm{C} 3$ & $1.473(9)$ & C10-C11 & $1.448(7)$ \\
\hline C1-H1A & 0.9700 & C11-C12 & $1.357(7)$ \\
\hline C1-H1B & 0.9700 & C11-C13 & $1.519(7)$ \\
\hline F-C16 & $1.364(6)$ & C12-C19 & $1.439(7)$ \\
\hline $\mathrm{C} 2-\mathrm{C} 3$ & $1.460(8)$ & C13-C14 & $1.373(8)$ \\
\hline $\mathrm{C} 2-\mathrm{H} 2 \mathrm{~A}$ & 0.9700 & C13-C18 & $1.375(8)$ \\
\hline $\mathrm{C} 2-\mathrm{H} 2 \mathrm{~B}$ & 0.9700 & $\mathrm{C} 14-\mathrm{C} 15$ & $1.391(8)$ \\
\hline C3-C4 & $1.496(8)$ & C14-H14A & 0.9300 \\
\hline C3-H3A & 0.9800 & C15-C16 & $1.361(8)$ \\
\hline $\mathrm{C} 4-\mathrm{C} 12$ & $1.422(7)$ & C15-H15A & 0.9300 \\
\hline $\mathrm{C} 5-\mathrm{C} 10$ & $1.414(7)$ & C16-C17 & $1.342(8)$ \\
\hline C5-C6 & $1.418(7)$ & C17-C18 & $1.390(8)$ \\
\hline $\mathrm{C} 6-\mathrm{C} 7$ & $1.345(8)$ & C17-H17A & 0.9300 \\
\hline C6-H6A & 0.9300 & C18-H18A & 0.9300 \\
\hline $\mathrm{C} 7-\mathrm{C} 8$ & $1.412(8)$ & C19-H19B & 0.9700 \\
\hline C7-H7A & 0.9300 & C19-H19C & 0.9700 \\
\hline Angle & Data $\left(^{\circ}\right)$ & Angle & Data $\left(^{\circ}\right)$ \\
\hline C4-N-C5 & $118.0(4)$ & C8-C9-H9A & 119.6 \\
\hline $\mathrm{C} 2-\mathrm{C} 1-\mathrm{C} 3$ & $60.5(5)$ & C10-C9-H9A & 119.6 \\
\hline C2-C1-H1A & 117.7 & C9-C10-C5 & $119.2(5)$ \\
\hline C3-C1-H1A & 117.7 & C9-C10-C11 & $124.6(5)$ \\
\hline C2-C1-H1B & 117.7 & C5-C10-C11 & $116.2(5)$ \\
\hline C3-C1-H1B & 117.7 & C12-C11-C10 & $119.2(5)$ \\
\hline H1A-C1-H1B & 114.8 & C12-C11-C13 & $121.9(5)$ \\
\hline $\mathrm{C} 1-\mathrm{C} 2-\mathrm{C} 3$ & $61.5(5)$ & C10-C11-C13 & $118.9(5)$ \\
\hline $\mathrm{C} 1-\mathrm{C} 2-\mathrm{H} 2 \mathrm{~A}$ & 117.6 & C11-C12-C4 & $119.5(5)$ \\
\hline $\mathrm{C} 3-\mathrm{C} 2-\mathrm{H} 2 \mathrm{~A}$ & 117.6 & C11-C12-C19 & $120.6(5)$ \\
\hline $\mathrm{C} 1-\mathrm{C} 2-\mathrm{H} 2 \mathrm{~B}$ & 117.6 & C4-C12-C19 & $119.9(5)$ \\
\hline $\mathrm{C} 3-\mathrm{C} 2-\mathrm{H} 2 \mathrm{~B}$ & 117.6 & C14-C13-C18 & $119.5(5)$ \\
\hline $\mathrm{H} 2 \mathrm{~A}-\mathrm{C} 2-\mathrm{H} 2 \mathrm{~B}$ & 114.7 & C14-C13-C11 & $120.1(5)$ \\
\hline C2-C3-C1 & $58.0(4)$ & C18-C13-C11 & $120.4(5)$ \\
\hline C2-C3-C4 & $120.3(6)$ & C13-C14-C15 & $120.2(6)$ \\
\hline $\mathrm{C} 1-\mathrm{C} 3-\mathrm{C} 4$ & $119.5(5)$ & C13-C14-H14A & 119.9 \\
\hline C2-C3-H3A & 115.6 & C15-C14-H14A & 119.9 \\
\hline $\mathrm{C} 1-\mathrm{C} 3-\mathrm{H} 3 \mathrm{~A}$ & 115.6 & C16-C15-C14 & $118.0(6)$ \\
\hline C4-C3-H3A & 115.6 & C16-C15-H15A & 120.1 \\
\hline N-C4-C12 & $123.3(5)$ & C14-C15-H15A & 120.1 \\
\hline $\mathrm{N}-\mathrm{C} 4-\mathrm{C} 3$ & 116.1(5) & C17-C16-C15 & $123.7(5)$ \\
\hline $\mathrm{C} 12-\mathrm{C} 4-\mathrm{C} 3$ & $120.6(5)$ & C17-C16-F & $118.8(6)$ \\
\hline $\mathrm{N}-\mathrm{C} 5-\mathrm{C} 10$ & $123.8(5)$ & C15-C16-F & $117.4(6)$ \\
\hline $\mathrm{N}-\mathrm{C} 5-\mathrm{C} 6$ & $117.9(5)$ & C16-C17-C18 & $117.9(6)$ \\
\hline C10-C5-C6 & 118.3(5) & C16-C17-H17A & 121.1 \\
\hline C7-C6-C5 & $121.7(5)$ & C18-C17-H17A & 121.1 \\
\hline C7-C6-H6A & 119.1 & C13-C18-C17 & $120.7(6)$ \\
\hline C5-C6-H6A & 119.1 & C13-C18-H18A & 119.6 \\
\hline C6-C7-C8 & $119.5(6)$ & C17-C18-H18A & 119.6 \\
\hline C6-C7-H7A & 120.3 & $\mathrm{C} 12-\mathrm{C} 19-\mathrm{Br}$ & $110.9(4)$ \\
\hline C8-C7-H7A & 120.3 & C12-C19-H19B & 109.5 \\
\hline C9-C8-C7 & $120.5(6)$ & Br-C19-H19B & 109.5 \\
\hline C9-C8-H8A & 119.8 & C12-C19-H19C & 109.5 \\
\hline C7-C8-H8A & 119.8 & Br-C19-H19B & 109.5 \\
\hline C8-C9-C10 & $120.8(5)$ & H19B-C19-H19C & 108.0 \\
\hline $\mathrm{C} 1-\mathrm{C} 2-\mathrm{C} 3-\mathrm{C} 4$ & $-107.9(7)$ & C10-C11-C12-C4 & $-0.1(8)$ \\
\hline C2-C1-C3-C4 & $109.3(7)$ & C13-C11-C12-C4 & $-177.8(5)$ \\
\hline C5-N-C4-C12 & $-0.7(8)$ & C10-C11-C12-C19 & $-179.5(5)$ \\
\hline C5-N-C4-C3 & 179.9(5) & C13-C11-C12-C19 & $2.8(8)$ \\
\hline C2-C3-C4-N & 34.6(9) & N-C4-C12-C11 & $0.7(9)$ \\
\hline C1-C3-C4-C12 & $147.2(7)$ & C3-C4-C12-C19 & $-0.5(8)$ \\
\hline $\mathrm{C} 4-\mathrm{N}-\mathrm{C} 5-\mathrm{Cl} 0$ & $0.1(8)$ & C12-C11-C13-C14 & $-101.2(6)$ \\
\hline C4-N-C5-C6 & $179.0(5)$ & C10-C11-C13-C14 & $81.1(7)$ \\
\hline N-C5-C6-C7 & $179.1(5)$ & C12-C11-C13-C18 & $76.5(7)$ \\
\hline C10-C5-C6-C7 & $-2.0(8)$ & C10-C11-C13-C18 & $-101.2(6)$ \\
\hline
\end{tabular}




\begin{tabular}{llll}
\hline C5-C6-C7-C8 & & & $-2.2(8)$ \\
C6-C7-C8-C9 & $1.1(9)$ & C18-C13-C14-C15 & $175.5(5)$ \\
C7-C8-C9-C10 & $0.3(10)$ & C11-C13-C14-C15 & $0.5(9)$ \\
C8-C9-C10-C5 & $-0.7(10)$ & C13-C14-C15-C16 & $1.6(10)$ \\
C8-C9-C10-C11 & $-0.2(9)$ & C14-C15-C16-C17 & $-178.5(5)$ \\
N-C5-C10-C9 & $179.8(5)$ & C14-C15-C16-F & $-1.9(9)$ \\
C6-C5-C10-C9 & $-179.6(5)$ & C15-C16-C17-C18 & $178.2(5)$ \\
N-C5-C10-C11 & $1.5(8)$ & -C16-C17-C18 & $1.9(9)$ \\
C6-C5-C10-C11 & $0.4(8)$ & C14-C13-C18-C17 & $-175.8(5)$ \\
C9-C10-C11-C12 & $-178.5(5)$ & C11-C13-C18-C17 & $0.1(9)$ \\
C5-C10-C11-C12 & $179.6(5)$ & C16-C17-C18-C13 & $-101.2(5)$ \\
C9-C10-C11-C13 & $-0.3(7)$ & C11-C12-C19-Br & $79.4(6)$ \\
C5-C10-C11-C13 & $-2.6(8)$ & C4-C12-C19-Br & \\
\hline Symmetry code: (i) x, y, z (ii) -x, -y, -z & $177.4(5$ & &
\end{tabular}

Symmetry code: (i) $\mathrm{x}, \mathrm{y}, \mathrm{z}$ (ii) $-\mathrm{x},-\mathrm{y},-\mathrm{z}$

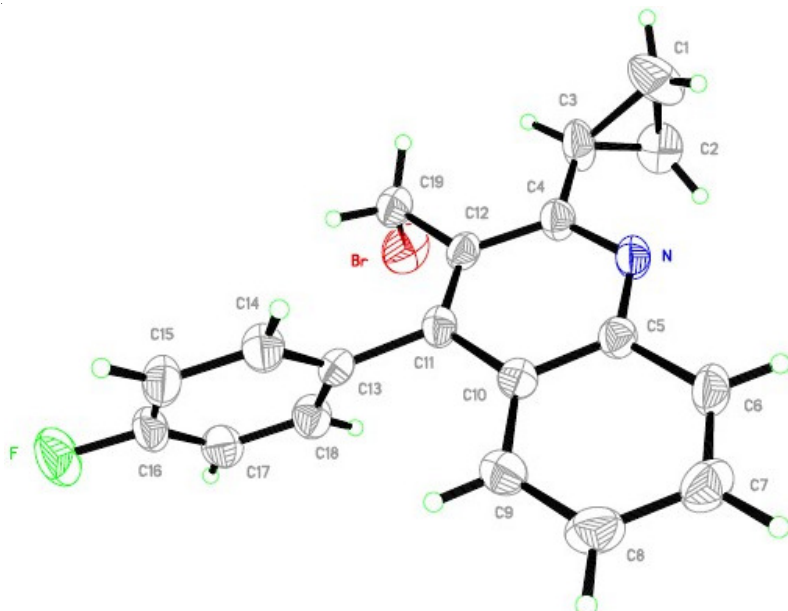

Fig. 3. General appearance of Compound I with the atoms represented by thermal vibration ellipsoids of $50 \%$ probability

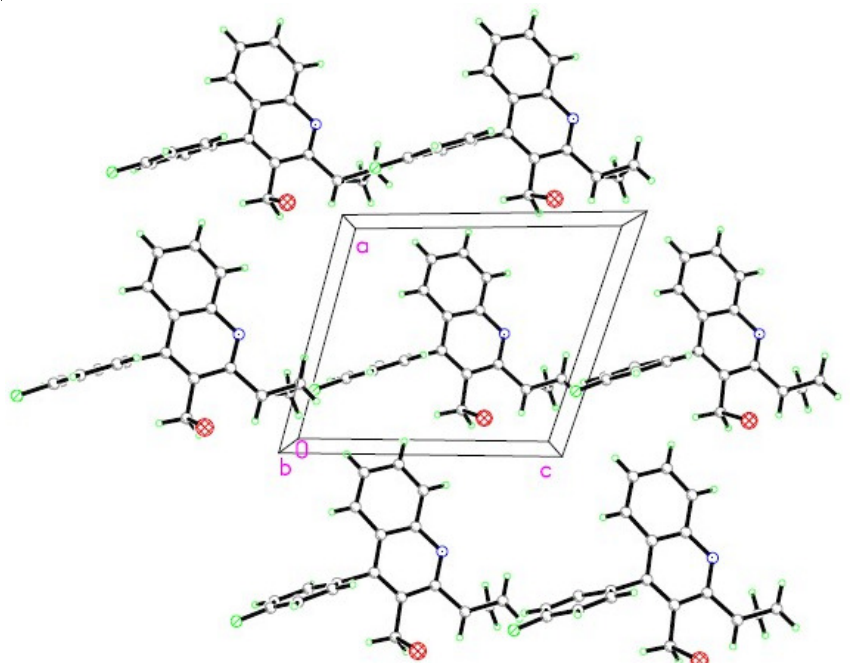

Fig. 4. Packing diagram for Compound $\mathbf{I}$
$=1.5 \mathrm{Ueq}(\mathrm{C})$ for other $\mathrm{H}$. There are no intramolecular and intermolecular hydrogen bonds in the structure. Unit cell parameters: $\mathrm{a}=9.6150(19), \mathrm{b}=9.868(2), \mathrm{c}=10.060(2) \AA, \mathrm{V}$ $=783.3(4) \AA^{3} ; Z=2$.

\section{ACKNOWLEDGEMENTS}

The authors gratefully acknowledge the support of Nanjing University and Changzhou University in analysis.

\section{REFERENCES}

1. E. Nicole and R. Yvonne, WO 2004103977 (2004).

2. C. Zdenko and S. Damjan, WO 2012013325 (2012).

3. F.H. Allen, O. Kennard, D.G. Watson, L. Brammer, A.G. Orpen and R. Taylor, J. Chem. Soc., Perkin Trans. 2, S1 (1987).

4. Enraf-Nonius, CAD-4 Software. Enraf-Nonius, Delft, The Netherlands (1985).

5. J. Folkman, Sci. Am., 275, 150 (1996)

6. K. Harms and S. Wocadlo, XCAD4. University of Marburg, Germany (1995).

7. A.C.T. North, D.C. Phillips and F.S. Mathews, Acta Crystallogr. A, 24, 351 (1968).

8. G.M. Sheldrick, Acta Crystallogr. A, 64, 112 (2008). 